\title{
Production of poly- $\beta$-hydroxybutyric acid (PHB) by Bacillus cereus on pineapple peels
}

\author{
Babaniyi Babfemi Raphael ${ }^{1}$ and Adelaja O A 2,* \\ ${ }^{1}$ National Biotechnology Development Agency. Abuja. Nigeria. \\ 2 Department of Chemistry, Federal University of Technology. Akure. Nigeria.
}

Publication history: Received on 28 June 2020; revised on 13 July 2020; accepted on 15 July 2020

Article DOI: https://doi.org/10.30574/gscarr.2020.4.1.0055

\begin{abstract}
Global population increase with the public demands could exceed the limit of natural resources soon and the current world problem on effective means of disposing used plastics which has posed great threat on the environment, therefore, this research was aim at producing biodegradable plastic using pineapple peels inoculated with Bacillus cereus. Sudan Black test was done to confirm the Bacillus cereus ability to produce polyhdroxybutyrate. Using fed-batch fermentation technology, polyhydroxybutyrate (PHB) pellets were produced, extracted and purified via solvent extraction. Pineapple peels as a substrate for the production of PHB yielded a good result with the cell dry weight of 4.7\%. The produced plastic was characterized using Fourier transform Infra-red (FT-IR) and gas chromatography mass spectroscopy (GC-MS). Bacillus cereus on pineapple peels can efficiently synthesized PHB which is a cheaper source and an alternative and better way to produced biodegradable plastic.
\end{abstract}

Keywords: Poly- $\beta$-hydroxybutyrate; Plastics; Biodegradable; Bacillus cereus; Pineapple peels.

\section{Introduction}

Plastics, known as synthetic polymer are used in a wide range of household, agricultural, marine and architectural applications as replacement to natural resources, such as metals and stones but its properties of durability have caused serious problems since plastic waste accumulates in the environment. Nature usually cannot handle plastic waste because plastics are not easily degraded by microorganisms [1]. Alternatives to waste disposal such as plastic recycling are not economical and are hazardous such as dioxin emission from PVC incineration [2]. Biodegradable plastics are designed to degrade under environmental conditions or in municipal and industrial biological waste treatment facilities [2]. There are different biodegradable polymer materials which one of them is Polyhydroxyalkanoates (PHAs) [3]. This polymer family is made of two major groups-aliphatic and aromatic. Polyhydroxyalkanoate (PHAs) are aliphatic polymer naturally produced via a microbial process on sugar-based medium, where they act as carbon and energy storage material in bacteria.

Among the PHA family is polyhydroxybutyrate (PHB). PHB is accumulated inside in numerous bacteria under nutrient - limiting conditions with excess carbon. Many researches have shown that number of microorganisms like Alcaligeneeutrophus, Azotobacterbeijierinckia, Pseudomonas Oleovorans, Rhizobium sp. and Bacillus sp. produce PHAs as reserve food material. They can be used in different applications such as packaging film and containers, surgical pins and sutures, and bone replacements. PHB degrade naturally and completely to $\mathrm{CO}_{2}$ and $\mathrm{H}_{2} \mathrm{O}$ under natural environment by different microorganisms $[4,5,6]$. Commercial production of PHB and its production from cheap and readily available as bio plastic as compared to that of production of synthetic plastics based on petrochemicals is a big challenge. Low cost agro industrial materials can be used to economize the production. Many efforts have been devoted to reducing the production costs by isolating bacterial strains capable of growing and producing PHB from inexpensive raw

\footnotetext{
${ }^{*}$ Corresponding author: Adelaja O A
} 
materials and also optimization fermentation conditions for PHB production [7]. Therefore, the objectives of this study was to isolate PHB- producing bacteria and characterize PHB produce on pineapple peels.

\section{Material and methods}

Fresh peels of pineapple were collected from Federal University of Technology, Akure. Pure strain of Bacillus cereus was obtained from research laboratory, Microbiology Departmental of Federal University of Technology, Akure. The Pineapple peels were washed with clean water and sun dried for 14 days. The dried peels were ground with electrical blender (VTcl 750 watts) and sieved with 32 mesh size sieve to have fine powders. The fine powders were carefully collected and kept in an air tight container for the production of polyhydroxybutrate.

\subsection{Sub-culturing of Bacillus cereus}

In order to preserve and to have enough of the organism, $2 \mathrm{~g}$ of nutrient agar (NA) was measured and $100 \mathrm{~mL}$ of distilled water was added in a flask and carefully shaken to allow homogenous dissolution of the mixture. The mixture was poured into three bottles (Biju bottle) $5 \mathrm{~mL}$ each and autoclave at $110 \circ \mathrm{C}$ for $15 \mathrm{~min}$. After sterilization, the bottles were removed from the autoclave and slant in a sterile condition to gel. The pure culture of Bacillus cereus was introduced to the prepared media with sterile loop and incubated for $24 \mathrm{hrs}$ at $37 \circ \mathrm{C}$, then kept in the refrigerator for later use.

\subsection{Confirmatory test (sudan black staining)}

Sudan black solution was prepared by dissolving $0.3 \mathrm{~g}$ of the powder in $100 \mathrm{~mL}$ of $70 \%$ ethanol. $2 \mathrm{~g}$ of nutrient agar was measured into a conical flask, $100 \mathrm{~mL}$ of distilled water was added and gently mixed, corked and autoclaved at $110 \circ \mathrm{C}$ for $15 \mathrm{~min}$. The media was poured into petri dish and allow to gel; then streaked with sterile loop, inoculated in a sterile condition and incubated for $24 \mathrm{hrs}$ at $37{ }^{\circ} \mathrm{C}$. The organism was smeared on grease free slide using sterile loop. The slide was allowed to dry, heat fixed, then stained for10 min. Excess sudan black dye was drained from the heat fixed slide: clarified with xylene blotted solution for 5s, reins in slow running tap water, air dried and viewed using X100 oil immersion lenses of celestron digital microscope [8].

\subsection{Seed culture preparation in nutrient broth for the production of polyhydroxybutyrate (PHB)}

The culture was prepared by dissolving $0.5 \mathrm{~g}$ of nutrient broth in $50 \mathrm{~mL}$ of distilled water, then heat with frequent agitation for one minute until complete dissolution. $7 \mathrm{~mL}$ was dispensed into test tube, corked and autoclaved at $110 \circ \mathrm{C}$ for $15 \mathrm{~min}$. After cooling, the broth was inoculated with Bacillus cereus in a sterile condition and incubated for $24 \mathrm{hrs}$ at $37 \circ \mathrm{C}$ with $120 \mathrm{rpm}$. The broth was centrifuge for $20 \mathrm{mins}$ at $4000 \mathrm{rpm}$. The supernatant was discarded and the organism (Bacillus cereus) settled at the base of the tube which was used for the production of polyhydroxybutyrate [9].

\subsection{Production of polyhydroxybutyrate (PHB)}

PHB was produced by preparing Kannan and Rehacek medium (Composition of media shown in table 1.0) [10]. With slight modification on pineapple peels as substrate. The substrate was soaked in $100 \mathrm{~mL}$ distilled water and kept at $4 \circ \mathrm{C}$ for $24 \mathrm{hrs}$. The substrate was sterilized separately at $110{ }^{\circ} \mathrm{C}$ for $10 \mathrm{~min}$ while the rest of the chemicals were sterilized at $121{ }^{\circ} \mathrm{C}$ for $15 \mathrm{~min}$. after cooling, they were all mixed together in erlenmeyer flask in a sterile condition and the pH adjusted to 6.8 using I N HCL and I M NaOH prior to the inoculation of the carbon source, the inoculum used was the fresh nutrient broth prepared in 2.3 above. The mixture was incubated for 48 hrs at $37 \circ \mathrm{C}$ with $120 \mathrm{rpm}$ the optical density, $\mathrm{pH}$ and rate of production of PHB were monitored 8 hourly by taken $50 \mathrm{~mL}$ aliquots and centrifuge at $4000 \mathrm{rpm}$ for the optical density, the frequency was set at $600 \mathrm{~nm}$ and curvet was properly cleaned for each of the reading.

Table 1 Composition of media

\begin{tabular}{llllll}
\hline Composition & $\begin{array}{l}\text { Yeast } \\
\text { Extract }\end{array}$ & $\begin{array}{l}\text { Potassium } \\
\text { Chloride }\end{array}$ & $\begin{array}{l}\text { Ammonium } \\
\text { Sulphate }\end{array}$ & $\begin{array}{l}\text { Pineapple } \\
\text { (Powder) }\end{array}$ & peels \\
\hline $\mathrm{g} / \mathrm{l}$ & 2.0 & 2.5 & 3.0 & 10.0 \\
\hline
\end{tabular}

\subsection{Extraction of PHA}

Extraction was done according to the methods described by Pooja 2014 and valappil et al., 2007 [9, 10] with a bit modification. After $48 \mathrm{hrs}$ of incubation, the cells were harvested. The slurry was poured into $50 \mathrm{~mL}$ centrifuging tube and centrifuged at $4000 \mathrm{rpm}$ for $20 \mathrm{~min}$, the supernatants were discarded, and the sediments were treated with $10 \mathrm{~mL}$ 
sodium hypochlorite solution and incubated again for $2 \mathrm{hrs}$ at $37^{\circ} \mathrm{C}$ with $120 \mathrm{rpm}$. The tubes were centrifuged again at 4000rpm for another $15 \mathrm{~min}$. this resulted into formation of three layers, first layer was the supernatants which contain sodium hypochlorite, that was discarded, the second layer was the layer having the PHB, the third layer contained the biomass resides which are heavier, the slurry were gently washed with $10 \mathrm{~mL}$ distilled water, acetone was added to further wash the remains of the PHB locked within the biomass, the tubes were stand for 10 min then decanted and 10 $\mathrm{mL}$ ice cold methanol was added to precipitates the dissolved PHB. $10 \mathrm{~mL}$ diethyl ether was added to further separate PHB from the biomass. Finally, $10 \mathrm{~mL}$ chloroform was added and the PHB float on the surface which were harvested with $44(2.5 \mu \mathrm{m})$ Whatman's filter paper. Weighed and oven dried at $28^{\circ} \mathrm{C}$ for one week. Cell dried weight of the composite were calculated using gravimetric method.

\subsection{Cell dry weight (CDW)}

This was done by gravimetric method by weighing the inertial weight of the harvested PHB and the final weight of the PHB. The PHB harvested was been weighed daily as it dried until constant weight was attained.

Cell dry weight $=\mathrm{W}_{\mathrm{o}}-\mathrm{W}_{\mathrm{f}}$

Where: $W_{o}$ is the inertial weight of PHB when it was produced

$\mathrm{W}_{\mathrm{f}}$ is the final weight of the PHB when it dried.

\subsection{Characterization of the polymer produced and standard (PHB)}

\subsubsection{Fourier transform infrared spectroscopy (FTIR)}

The dried sample of the polymer produced (PHB) $2 \mathrm{mg}$ was used to prepare KBr discs. An FT-IR Spectrum $1720 \mathrm{X}$ Spectrometer. The analysis was done with window material, csl, 10 scan, resolution $4 \mathrm{~cm}-1$, and spectral range $4400-$ $350 \mathrm{~cm}-1$. The detector was a temperature- stabilized coated DTGs detector. This analysis was done at central laboratory, university of Ibadan Nigeria.

\subsubsection{Gas chromatography- mass spectroscopy (GC-MS)}

Identification of polymer produced was done using GC-MS analysis of the methanolysed polymer. A quantity of $0.5 \mathrm{~g}$ of sample and $5 \mathrm{~mL}$ ethylbenzoate in chloroform $5 \mathrm{~mL}$, was added to a mixture of $3 \mathrm{~mL}, 15 \%$ Sulphuric acid in methanol 5 $\mathrm{mL}$ (ratio $1: 1$ ) at $100^{\circ} \mathrm{C}$ for $4 \mathrm{hrs}$ reflux. The reaction was left over night, $10 \mathrm{~mL}$ distilled water was added and the tube was vortexed for $2 \mathrm{~min}$, after phase separation, the organic phase was collected and dried over anhydrous magnesium sulphate. This was filtered and sent for GC-MS analysis at central laboratory. Afe Babalola University (ABUAD) AdoEkiti, Ekiti State, Nigeria. The analysis was done using Agilent 1909IS-933H-1MS. The sample in chloroform (1ul) was injected with helium $\left(1 \mathrm{ml} \mathrm{min}^{-1}\right)$ as the carrier gas. The injector temperature was $290{ }^{\circ} \mathrm{C}$ and the column temperature was increased from $30^{\circ} \mathrm{C}$ to $350{ }^{\circ} \mathrm{C}$ at $60^{\circ} \mathrm{C} \mathrm{min}-1$ and held at the final temperature for 10 min run time $27.333 \mathrm{~min}$.

\section{Results and discussion}

\subsection{Bacillus cereus confirmatory test (sudan back staining)}

Figure 1 shows the result of the sudan back staining of the Bacillus cereus. Sudan black stained Bacillus cereus appeared as gram positive rod shaped cells under oil immersion which was similar to the sudan back staining result of Nasir et al. 2016 [11] on production and characterization of Polyhydroxybutyrate (PHB). The positive strains were isolated and inoculated in the broth and prepared in nutrient broth for the production of polyhydroxybutyrate (PHB) in a sterile condition for further studies. 


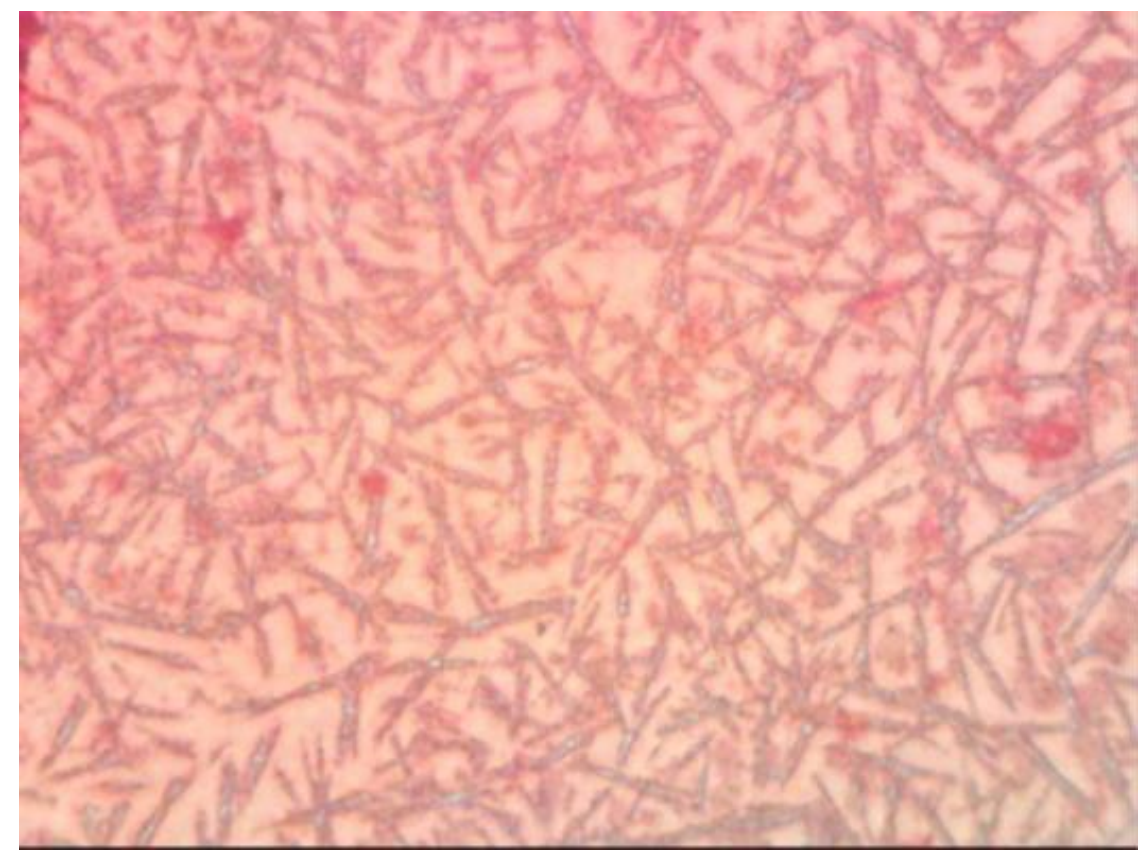

Figure 1 Bacillus cereus stained with Sudan black

\subsection{PHB production from the substrate}

The PHB production yield result of the pineapple peels is shown in Figure 2. Although, been moderately deferent to many observations recorded by different researchers, product yield increased as the media $\mathrm{pH}$ changed from neutral conditions to acidic condition. Figure 3 revealed changes in optical density (OD) and pH of the substrate during $48 \mathrm{hrs}$ of incubation. Higher product was obtained from the pineapple $(0.37 \mathrm{~g})$ at $\mathrm{pH}$ value of 4.8 , optical density of 1.902 and $48 \mathrm{hrs}$. And the PHB settles at the top of the tube which was also different from observations of previous researchers like pooja $[9,12]$. They reported that the PHB settles at the base of the tube. These observations may be due to the difference in carbon sources or the method used. The total dry cell at the end of the fermentation was 4.7 which was a good yield also showed that the Bacillus cereus used up the substrate effectively.

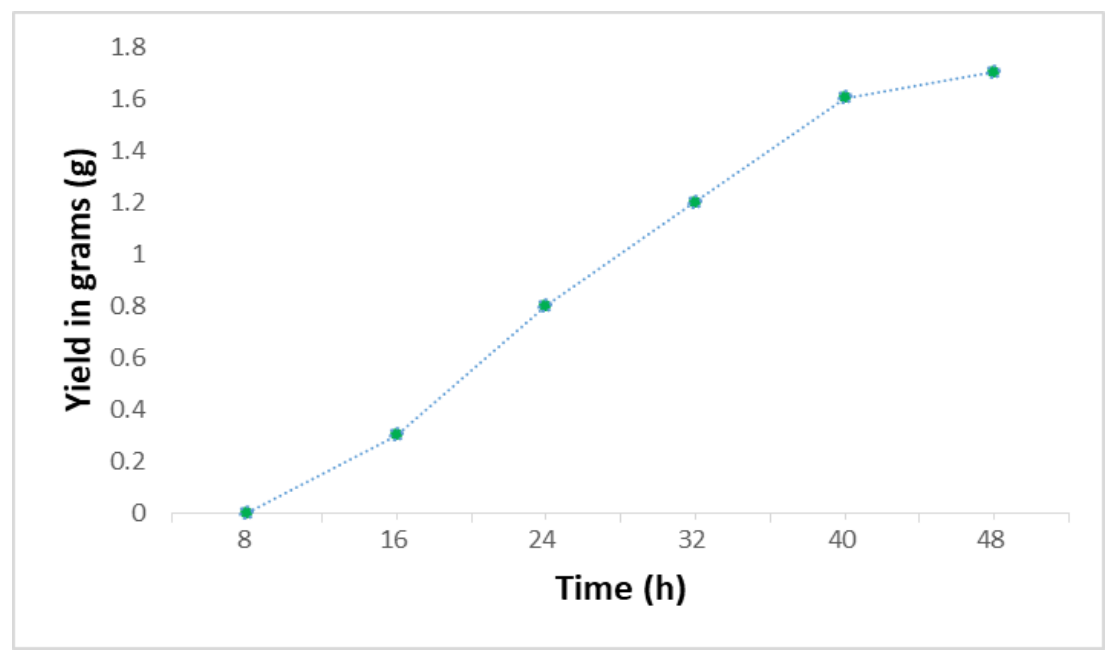

Figure 2 Substrate yield during incubation 


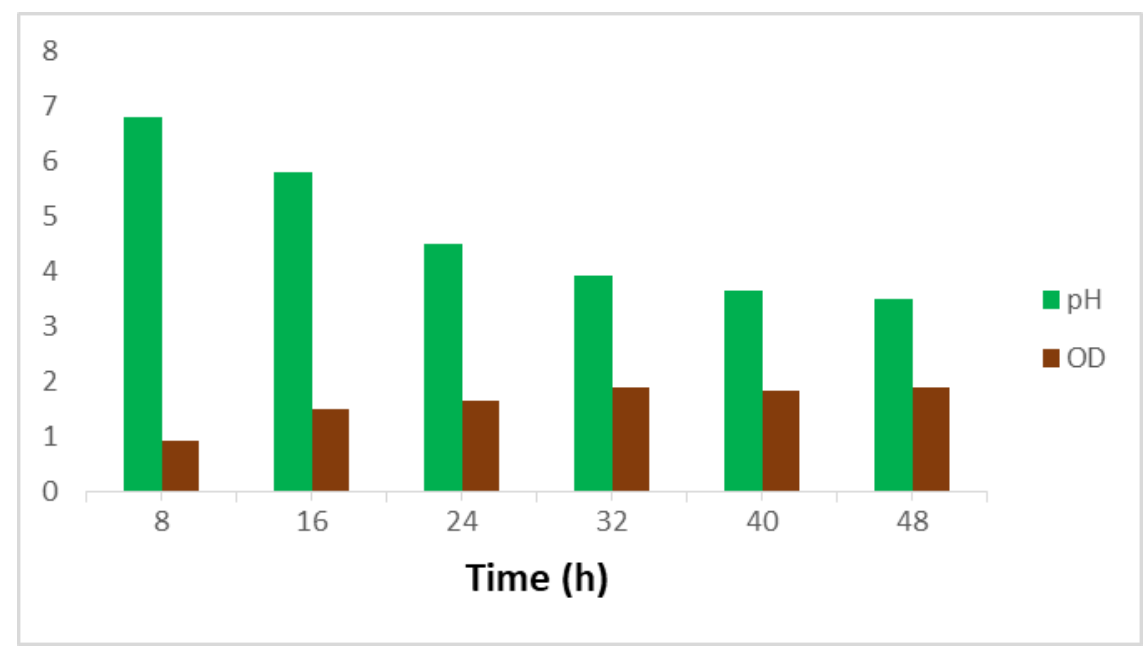

Figure $3 \mathrm{OD}$ and $\mathrm{pH}$ of the substrate during incubation period

\subsection{Characterization of the produced polymer}

\subsubsection{Fourier transform infrared spectroscopy (FT-IR) analysis}

The FT-IR characterization of the polymers is shown in Figure 4. The polymers extracted for pineapple peels showed the intense absorption at $3405 \mathrm{~cm}^{-1}$ were attributed to the terminal $\mathrm{O}-\mathrm{H}$ bonding or water adsorption on the PHB. Adsorption bands at $2949 \mathrm{~cm}^{-1}$ for $\mathrm{C}-\mathrm{H}$ stretching group were observed in the produced polymer corresponds to the stretching and deformation vibrations of the $0-\mathrm{H}$ groups. The presence of the peak above $2900 \mathrm{~cm}^{-1} \mathrm{may} \mathrm{be} \mathrm{due} \mathrm{to} \mathrm{the}$ $\mathrm{C}-\mathrm{H}$ hydrogen bond $[13,14]$. IR spectra of the polymers revealed the presence of marked peaks at wave numbers 1643 $\mathrm{cm}^{-1}$ representing the presence ester carbonyl $(\mathrm{C}=0)$ stretching groups, band recorded at $1022 \mathrm{~cm}^{-1}$ is generally known to be typical characteristics of sugar derivatives such as guluronic acid, manuronic acid and uronic acid.

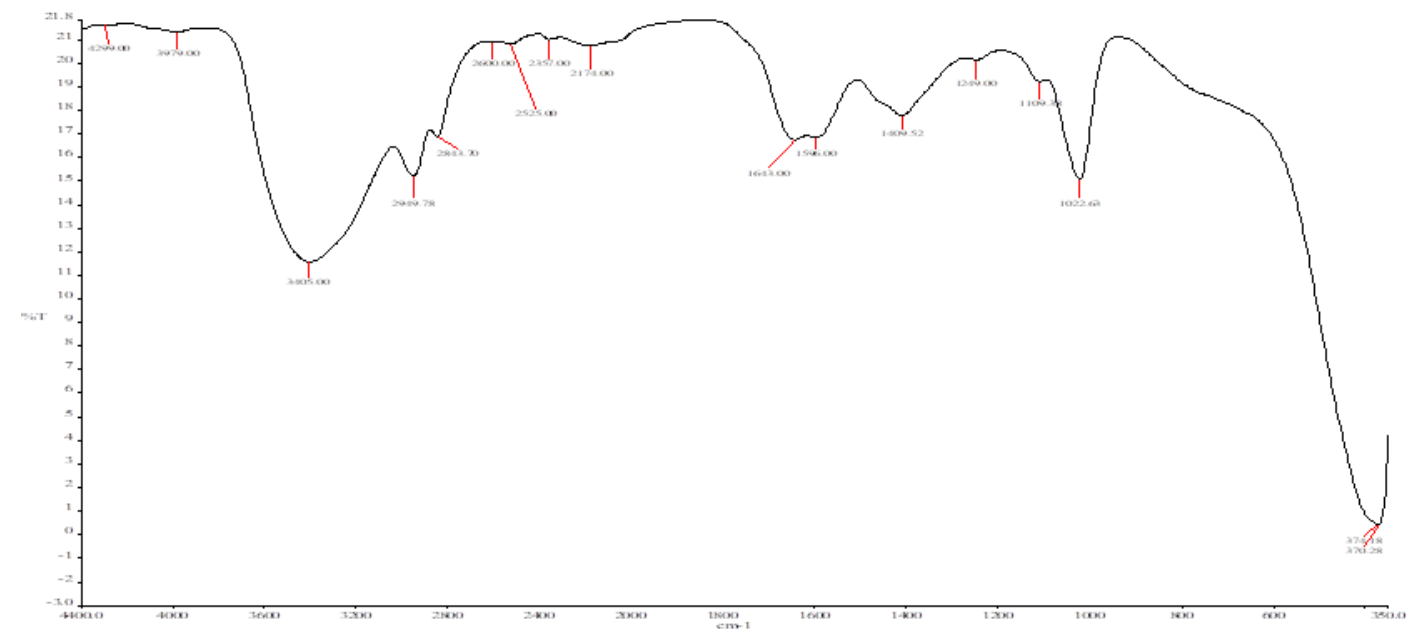

Figure 4 The FT-IR result of the PHB produced from pineapple peels.

\subsection{Gas chromatography-mass spectroscopy (GC-MS) analysis}

The result of the GC-MS analysis is shown in fig.5 below, the analysis elucidated the characteristic fragmentation patterns, suggesting the presence of PHB. The carbonyl and hydroxyl ends of the corresponding hydroxybutyrate were identified from the specific peaks in the chromatogram. The fragmentation patterns were in concordance with the results given by Mohan et al. (2010). The mass spectra for propyl-esters of 3-hydroxyalkanoates corresponding 3HB peaks with RT values of 10.62 corresponding to derivatized products of Butenoic acid confirming the presence of polyhydroxybutyrate. 


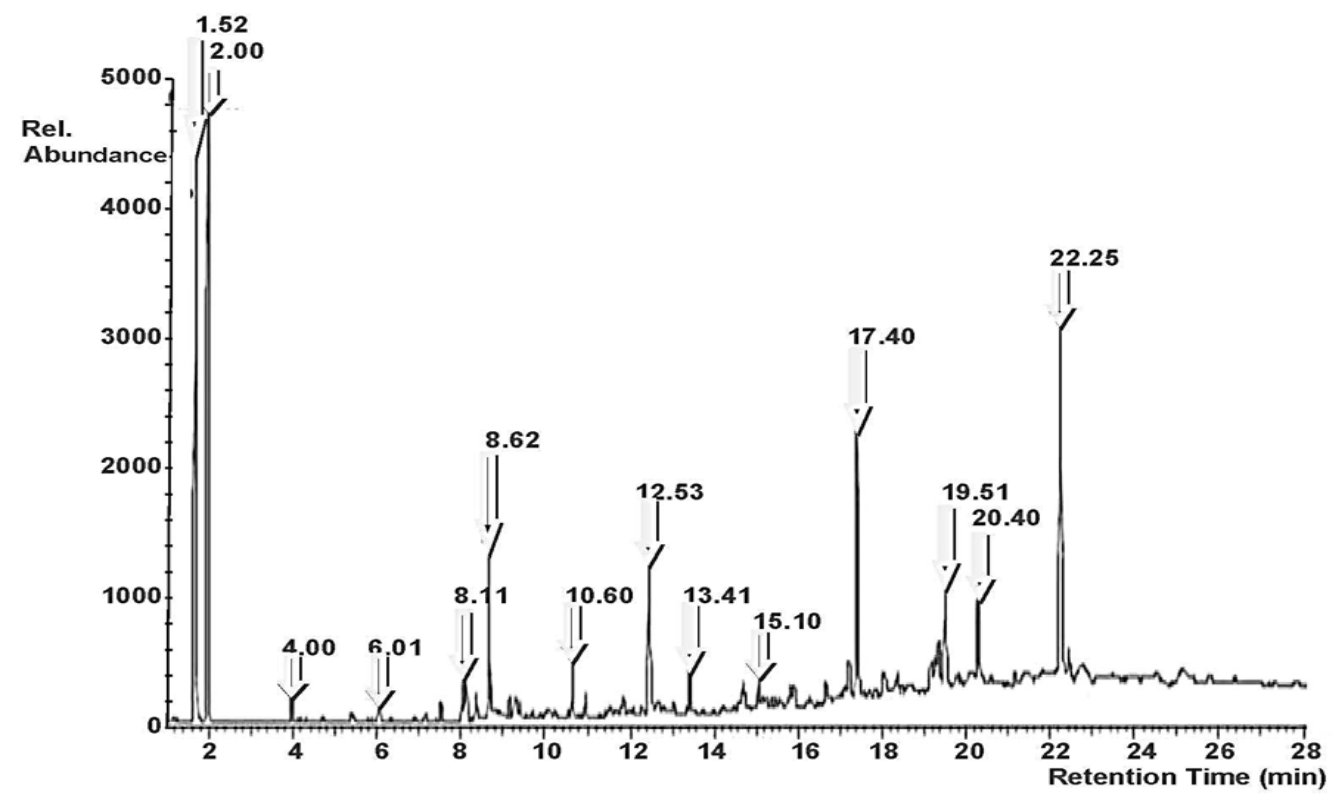

Figure 5 GC-MS analysis of PHB extracted from pineapple peels

\section{Conclusion}

The research has shown that the substrate (Pineapple peels) that was used for the production of PHB using Bacillus cereus are effective with a good yield. It was noted that during the production, there was increase in optical density with yield and decrease in the $\mathrm{pH}$ of the media. FT-IR and GC-MS analysis further affirmed that it was PHB that was produced. However, PHB production using pineapple peels with Bacillus cereus can be optimized for medical applications in future study.

\section{Compliance with ethical standards}

\section{Acknowledgments}

I Acknowledged staff and technologists of Chemistry and Microbiology departments, Federal University of technology. Akure.

\section{Disclosure of conflict of interest}

No conflict.

\section{References}

[1] Anon T, Sudarat S and Sutticha Na-Ranong T. (2017).Efficient polyhydroxybutyrate production from Bacillus thuringiensis using sugarcaneJuice substrate. Turk Journal of Biol, 41, 992-1002.

[2] Belal EB. (2013). Production of Poly- Hydroxybutyric Acid (PHB) by Rhizobium elti and Pseudomonas stutzeri. Curr. Res. Journal of Biol. Sci, 5(6) 273 - 284.

[3] Zhang S, Kolvek S, Lenz RW and Goodwin S. (2003). Mechanism of the polymerization reaction initiated and catalyzed by the polyhydroxybutyrate synthase of Ralsotonia eutropha. Biomacr, 4, 504-509.

[4] Yu H, Yin J, Li H, Yang S and Shen Z. (2000). Construction and selection of the novel recombinant Escherichia coli. Eur. J. Biosci. Bioeng, 89, 307-311.

[5] Morita M, Tanji Y, Mizoguchi K, Soejima A, Orito Y and Unno H. (2001). Antibacterial activity of Bacillus amyloliquefaciens phage endolysin without holing conjugation. J. Biosci. Bioeng, 91, 469-473.

[6] Singh P and Parmar N. (2011). Isolation and characterization of two novel polyhydroxybutyrate (PHB)-producing bacteria. Afr. J. Biotec, 10, 4907- 4919. 
[7] Kulpreecha S, Boonruangthavorn A, Meksiriporn B and Thongchul N. (2009). Inexpensive fed-batch cultivation for high poly(3-hydroxybutyrate) production by a new isolate of Bacillus megaterium. J. Biosc. Bioeng, 107(3), 240-245.

[8] Khadeejah NKO, Shittu KO and Kabiru AY. (2016). Production and characterization of polyhydroxyalkanoate (PHA) using mango seed kernel as an alternative to glucose. Biotechnology Journal International, 1-11.

[9] Pooja B. (2014). Biosynthetic of polyhydroxyalkanoate novel blends and composite application, 45-67.

[10] Valappil S, Boccaccini A, Bucke C and Roy I. (2007). Polyhydroxyalkanoates in Grampositive bacteria: insights from the genera Bacillus and Streptomyces. Antonie van Leeuwenhoek, 91, 1-17.

[11] Nasir-Naeem Ko, Shittu KO and Kabiru Ay. (2016). Production and characterization of PHA. Using mango seed kernel as an alternative to glucose. Biotechnology Journal international, 1-11.

[12] Mukai K and Doi Y. (1995). Microbial degradation of polyesters. Prog. Ind. Microbiol, 32, $189-204$.

[13] Mohan SV, Reddy MV, Subhash GV and Sarma PN. (2010). Fermentative effluents from hydrogen producing bioreactor as substrate for poly $(\beta-\mathrm{OH})$ butyrate production with simultaneous treatment: An integrated approach. Bioresource technology, 101(23), 9382-9386.

[14] Hahn SK, Chang YK and Lee SY. (1995). Recovery and characterization of poly (3-hydroxybutyric acid) synthesized in Alcaligenes eutrophus and recombinant Escherkichia coli. Appl. Environ. Microbiol, 61(1), 34-39.

\section{How to cite this article}

Babaniyi BR and Adelaja OA. (2020). Production of poly- $\beta$-hydroxybutyric acid (PHB) by Bacillus cereus on pineapple peels. GSC Advanced Research and Reviews, 4(1), 24-30. 